\title{
A PREVISÃO DO CONSUMO DE ELETRICIDADE NAS RESIDÊNCIAS DA CIDADE DE MAPUTO
}

\section{ARTIGO ORIGINAL}

CHAPALA, Nelson Manuel Alfredo ${ }^{1}$

MAÚRE, Genito Amós ${ }^{2}$

SILVA, Carlos ${ }^{3}$

CHAPALA, Nelson Manuel Alfredo. MAÚRE, Genito Amós. SILVA, Carlos. A previsão do Consumo de Eletricidade nas residências da Cidade de Maputo. Revista Científica Multidisciplinar Núcleo do Conhecimento. Ano 05, Ed. 10, Vol. 21, pp. 0523. Outubro de 2020. ISSN: 2448-0959, Link de acesso: https://www.nucleodoconhecimento.com.br/engenharia-eletrica/consumo-deeletricidade

\section{RESUMO}

Com a crescente utilização de equipamentos elétricos nas residências, em parte resultante do aumento exponencial do número e da melhoria das condições de vida da população, tornou-se necessário analisar a demanda de eletricidade em residências. E neste artigo é estudado o quadro teórico da demanda de eletricidade no setor residencial da Cidade de Maputo. Para isso, é analisado o consumo de equipamentos elétricos das 577 residências, 469 suburbanas e 108 urbanas, e pela utilização das elasticidades equipamento $\mathrm{x}$ consumo é projetado o consumo futuro de

\footnotetext{
${ }^{1}$ Mestrado em Informática e Graduado em Engenharia Eletrotécnica. Estudante de Doutoramento, Centro de Pesquisa em Energia, Universidade Eduardo Mondlane, Moçambique.

2 Doutoramento em Ciências Ambientais e Geográficas.

${ }^{3}$ Doutorado em Engenharia Mecânica.
} 
eletricidade nestas residências. Os resultados indicam que os equipamentos mais elásticos nas residências são as lâmpadas incandescentes, e um aumento de $12 \%$ na penetração de lâmpadas incandescentes de $60 \mathrm{~W}$ nas famílias suburbanas, o consumo anual por família pode aumentar em $80 \mathrm{KWh}$ e 18GWh em todas residências suburbanas. E um aumento de $1 \%$ de lâmpadas fluorescentes nas residências urbanas pode fazer com que o consumo anual por família aumente em 1,9KWh.

Palavras-chave: Residências, consumo, demanda, elasticidade, eletrodomésticos.

\section{INTRODUÇÃO}

O aumento do consumo de energia ocorrido nos últimos anos tem sido atribuído à crescente urbanização, aumento da temperatura e da renda monetária das famílias e a dependência de energias, como o gás e a eletricidade (SILVA, 2017; REDDY, 1994). No caso da eletricidade, muitas vezes o aumento do consumo nas residências é atribuído ao crescimento do número de equipamentos eléctricos (SILVA, 2017; LAICANE et al., 2015; REDDY, 1994; BENZERA, 2017).

Trata-se de equipamentos que sem dúvida contribuem bastante para o pico do sistema (ZARNIKAU, 2003). Diante destes acontecimentos, torna necessário conhecer como os diferentes serviços domésticos, tais como a refrigeração ou iluminação, contribuem para a saturação da curva de carga no momento do pico e também em outras horas do dia. E neste contexto, já existem tantas abordagens, sendo que algumas delas preveem a evolução do consumo com a utilização de dados demográficos e da taxa de atendimento (SILVA, 2017). No caso da cidade de Maputo, refira-se, com a taxa de atendimento máxima (eletrificação à 100\%) e o número populacional anual quase constante, o consumo nas residências só pode ser interpretado com base no consumo de cada eletrodoméstico.

A questão central deste estudo é, em que medida o crescente uso de equipamentos eléctricos nas residências afeta o consumo de eletricidade em Maputo. As outras questões que são colocadas são: Qual é o equipamento eléctrico que mais contribui para a saturação da curva da carga na hora de pico? Quanta a energia pode ser RC: 62631

Disponível em: https://www.nucleodoconhecimento.com.br/engenharia-eletrica/consumo-deeletricidade 
poupada com as alterações de natureza tecnológica dos equipamentos atualmente em uso?

Para responder-se estas questões, é preciso conhecer o consumo de energia de forma mais decomposta, ou para cada equipamento em uso nas residências (PARTI e PARTI, 1980). Para isto, uma ferramenta fundamental proposta neste artigo é Análise condicionada da demanda, com a utilização da elasticidade. A principal contribuição deste estudo é determinar a importância de alguns eletrodomésticos no sector residencial da Cidade de Maputo na carga eléctrica. $O$ estudo analisa os dados em nível micro, usando a abordagem do censo de aparelhos para estimar o consumo de aparelhos usados nas residências, para depois relacionar o uso de eletricidade à mudanças nos níveis de penetração de aparelhos. As elasticidades dos aparelhos estimadas neste estudo permitem fazer uma previsão do consumo futuro de eletricidade, e os resultados podem ser utilizados para o planeamento do mercado de eletricidade, no direcionamento dos programas e/ou as campanhas de racionalização do uso racional de energia ou campanhas publicitárias.

\section{A UtilizaçÃo de elasticidade para A pREVisão de CONSUMO DE ELECTRICIDADE EM RESIDÊNCIAS}

Um estudo de referência para este tipo de abordagem remonta desde 1971 (de Wilson). Wilsson (1971) propôs o modelo econométrico para o cálculo da elasticidade dos equipamentos através do seguinte modelo:

$$
Q_{t}=\kappa+\beta_{1} P_{t}+\beta_{2} G_{t}+\beta_{3} Y_{t}+\beta_{4} R_{t}+\beta_{5} C_{t}+\varepsilon \text { (na sua forma linear) }
$$

Onde: "K" é o valor de origem da função de demanda; "Qt" é o logaritmo natural da quantidade da energia eléctrica que é consumida por cada residência; " $\mathrm{Pt}_{\mathrm{t}}$ é o logaritmo natural do preço/tarifa de energia eléctrica; "G" é o logaritmo natural de preço médio do gás natural; "Y" é o logaritmo natural da renda residencial; " $\mathrm{R}_{\mathrm{t}}$ " é o logaritmo natural de quartos de cada residência; "C $\mathrm{C}_{t}$ é o logaritmo natural de 
aquecimento ou temperaturas diárias; " $\varepsilon$ " é o termo de erro de regressão ou com pressuposições usuais; O parâmetro " $\beta$ " representa as elasticidades da procura de energia eléctrica em relação à cada caso da equação 1 .

O modelo econométrico da procura residencial de eletricidade de Wilson (1971) também foi estimado na forma logarítmica (equação 2), com a finalidade de obter-se diretamente a elasticidade (GARCEZ e GHIRARDI, 2005).

$$
\ln Q=10.25-1.3 \ln P+0.3 \ln G-0.46 \ln Y+0.49 \ln R+0.04 \ln C
$$

$\mathrm{Na}$ equação (2), apesar da renda ser negativa (Y), Wilson (1971) encontrou o sinal esperado em relação ao preço, cuja justificativa baseia-se na escolha dos dados de corte e na representação de uma função de procura de longo prazo (GARCEZ e GHIRARDI, 2005). O resultado da equação 2 será positivo ou favorável ao consumidor, se o seu consumo de eletricidade aumentar em função do aumento da sua renda.

O modelo de Wilson (1971) depois foi aprimorado por Anderson (1973), que utilizou a função 3 para determinar a procura (demanda) da elasticidade. Relativamente ao modelo de Wilson (1971), Anderson (1973) incorporou os preços do óleo para aquecimento $(P O)$ e de carvão $(P C)$, o número de residências não urbanas $(N U)$ e o tamanho médio da família $(H S)$.

$$
\begin{aligned}
& \ln X=a_{0}+a_{1} \ln P E+a_{2} \ln P G+a_{3} \ln P O+a_{4} \ln P C+a_{5} \ln P B G+a_{6} \ln Y+a_{7} \ln H S+ \\
& a_{8} S H U+a_{9} N U+a_{10} W+a_{11} S+u
\end{aligned}
$$

Os outros parâmetros utilizados no modelo de Anderson são: o consumo residencial de eletricidade $(X)$; o preço de eletricidade $(P E)$; o preço médio do todo tipo de gás $(P G)$; o preço do gás de cozinha $(P B G)$; a renda familiar ( $Y$; quantidade de eletrodomésticos ou equipamentos residenciais ( $\mathrm{SHU}$ ); a temperatura média de Dezembro $(W)$; a temperatura média de Julho $(S)$; e o termo de erro da regressão “ u". 
Em relação ao modelo de Wilson (1971), pode-se observar que Anderson (1973) também incluiu as variáveis climáticas ( $W$ e $\mathrm{S}$ ), mas em função dos meses em que registam-se os défices ou a maior procura. De resto, na equação 3 são utilizadas as principais variáveis utilizadas no modelo convencional de demanda da eletricidade, nomeadamente o preço da eletricidade e renda familiar (GARCEZ e GHIRARDI, 2005). Como sempre, é possível denotar que a renda familiar e o preço ou tarifa de energia são as variáveis principais para este tipo de análise. E as outras variáveis vão sendo incorporadas se realmente influenciarem de forma significativa nos hábitos de utilização dos equipamentos, ou mesmo no consumo, ou na renda, das famílias. Como exemplo, a incorporação da função " $P G$ " no modelo de Anderson (1973) só cria uma complexidade desnecessária ao modelo, pois é o gás de cozinha que contribui mais nas rendas das famílias.

O terceiro estudo destacável é de autoria de Parti e Parti (1980), que analisaram a procura de energia eléctrica em residências, também da Califórnia. Os autores propuseram a utilização da regressão estatística para quebrar o consumo de cada equipamento eléctrico residencial da Califórnia. Na década 80 foram surgindo diversas abordagens em torno destes modelos, tanto para estimar o consumo assim como analisar a demanda horária de cada residência ou equipamento, e destacam-se os estudos de Archibald et al. (1982), Garbacz (1983), Aigner et al. (1984), Donnelly (1984) e Stanovnik (1987).

Enquanto nas últimas três décadas, igualmente surgiram muitos estudos e um deles é de Mattos e Lima (2005). Mattos e Lima (2005) basearam-se em modelos top-down econométricos (função 4), onde utilizaram a tarifa média de energia.

$$
Q_{t}=\alpha+\beta_{1} R_{t}+\beta_{2} T_{t}+\beta_{3} E L_{t}+v_{t}
$$

Na equação 4, " $\alpha$ " foi definido como valor de origem da função de procura; "Q" é o logaritmo natural da quantidade de energia eléctrica que é consumida por cada residência no tempo $t$; " $\mathrm{R}_{\mathrm{t}}$ " é o logaritmo natural da renda residencial no tempo $t$; " $\mathrm{T} t$ " é o logaritmo natural da tarifa residencial de energia eléctrica no tempo $t$, "ELt" é o 
logaritmo natural do preço dos eletrodomésticos no tempo $t$, "Vt" é o termo de erro (com pressuposições usuais); " $t$ " é o tempo (medido em anos); Os parâmetros " $\beta_{1}$ ", " $\beta_{2}$ " e " $\beta_{3}$ " são as elasticidades da procura de energia eléctrica em relação à renda, ao preço de energia eléctrica e ao preço dos eletrodomésticos, respectivamente.

Da equação 4 , com $\beta_{1}>0$, o consumo de energia eléctrica em residências aumenta com o aumento da renda dos consumidores. E o consumo vai reduzir se for incrementada a tarifa de energia eléctrica e do preço dos eletrodomésticos $\left(\beta_{2}, \beta_{3}>0\right)$. O primeiro caso é considerado positivo, pois aceita-se que o consumo de energia eléctrica em residências aumente com o aumento de renda dos consumidores, e o segundo caso é desfavorável ao consumidor, uma vez que o seu consumo aumentará em função do aumento das tarifas e dos preços dos eletrodomésticos.

Entretanto, embora seja uma prática comum usar elasticidades de preço para prever a demanda futura de energia, devido ao subsídio oculto nos preços da energia é difícil estimar as elasticidades (TAYLOR, 1975). Repare-se que mesmo nos países em que muita pesquisa sobre demanda de eletricidade foi realizada, pouco se sabe sobre as elasticidades de preços e o tempo que demora nas respostas à demanda (SILVA, 2017).

Com isto, Dubin e McFaden (1984) mostram que para obter uma ideia realística da demanda de eletricidade por diferentes serviços residenciais, é fundamental considerar a composição do número de eletrodomésticos. Essa composição pode ser influenciada pela renda familiar, tamanho da família, etc. (PARTI e PARTI, 1980). E é com base neste pensamento ou limitações que Reddy (1994) propôs a utilização de elasticidade equipamento $x$ consumo para prospecção do consumo em residências. Reddy (1994) utilizou dados de uma pesquisa residencial para estimar o consumo de eletricidade em diferentes equipamentos, relacionando as estimativas de consumo e as elasticidades obtidas por equipamento. É através dessas estimativas e das variações no estoque de equipamentos que o autor projetou o mercado futuro de energia para o município de Bangalore, Cidade sul-indiana. 
Reddy (1994) propôs a utilização da elasticidade no consumo de energia domiciliar, onde:

- " $\mathrm{X}_{\mathrm{i}}$ " foi definido como o número de equipamento da "ja" categoria em uma residência, e " $X_{j}$ " o número total de eletrodomésticos/equipamentos eléctricos da "ja" categoria em todas as "N" residências, ou seja:

$$
X_{j}=\sum X_{i j}
$$

- Definiu-se o termo "X" como sendo o número médio de equipamentos da "ja" categoria por residência, neste estudo denominado como coeficiente de penetração. Ou seja:

$$
\mathrm{X}=\frac{X_{j}}{N}
$$

- O termo "E" foi definido como o consumo total das residências estudadas, e o consumo médio (da residência modelo) foi definido pela função:

$$
E_{M}=\frac{E}{N}
$$

Entretanto, se o parque " $\mathrm{Xj}_{\mathrm{j}}$ " de equipamentos da "ja" categoria for aumentado por uma unidade, isto é, um equipamento desta categoria for adicionado ao parque de equipamentos da residência "R", então a mudança percentual do número de um certo equipamento na residência é (REDDY, 1994):

$$
E=\frac{(100)}{X_{j}}
$$


Em resposta a este estímulo, o consumo mensal de energia eléctrica em cada residência aumenta em "Bj" e a mudança ou aumento percentual do consumo da electricidade de um certo equipamento nesta residência é (REDDY, 1994):

$$
B_{j} X_{j}=\frac{\left(100 X_{j}\right)}{E}
$$

A razão destes acréscimos percentuais (8 e 9), Reddy (1994) designou de elasticidade " $\eta$ ", representado pela seguinte expressão matemática:

$$
\eta=\frac{B_{j} X_{j}}{E}
$$

Na equação 10, na medida em que os equipamentos pouco eficientes são substituídos por mais eficientes, as elasticidades-equipamento irão decrescer automaticamente.

Estas elasticidades permitem ordenar os equipamentos segundo seu impacto sobre a demanda e, assim, priorizá-los em programas de gestão ou em campanhas de educação e sensibilização para o uso racional (REDDY, 1994). Reddy (1994) determinou os impactos do crescimento do parque de cada equipamento sobre o consumo residencial do município de Bangalore. Outrossim, Reddy (1994) estudou as participações de cada uso final no consumo total e fez algumas inferências sobre o potencial de racionalização em alguns equipamentos mais importantes nas residências de Bangalore.

\section{METODOLOGIA}

No presente estudo é adoptado o raciocínio de Reddy (1994). Por um lado porque nas residências de Maputo ainda podem ser utilizados novos equipamentos (diferentes dos registados), e por outro porque a evolução do consumo nas residências, pelo menos até em 2030, vai depender do consumo de eletrodomésticos (CHAPALA, trabalho em curso). 
Considerando " $X$ ", o número total de equipamentos da categoria "j" em todos os bairros suburbanos (BS) ou urbanos (BU), e "N", o número total de residências também em todos os bairros suburbanos ou urbanos, obteve-se o número médio dos equipamentos $(£)$ da categoria "j" em cada residência do grupo (BS ou BU), pela expressão 6.

As elasticidades equipamento - consumo " $\eta$ " são obtidas pela equação 10. Tal como o estudo de Redy (1994) foi considerado como referência o consumo médio por família. Para isso, no cálculo de elasticidade foi considerado o consumo médio de uma casa urbana ou suburbana, determinado de acordo com a expressão 7 .

Na equação 10, " $E$ " é o consumo médio de uma família suburbana ou urbana, " $B$ " é o coeficiente de penetração $(£)$ e " $X_{j}$ " é a participação (em KWh) de equipamento da categoria "j" no consumo médio de uma casa urbana ou suburbana. (vide no Quadro 1). De salientar que as elasticidades consumo - equipamento são válidas em situações que as residências não estão saturadas, como as da Cidade de Maputo, ou seja, em situações em que todas as residências têm a capacidade para receber mais equipamentos eléctricos (vide os coeficientes de penetração dos equipamentos no Quadro 1).

Para a projeção do consumo da zona urbana da Cidade de Maputo, os resultados da equação 10 (para cada tipo de equipamento) são multiplicados por 20352 flats e multiplicados por 224653 para obter-se a projeção do consumo nas residências suburbanas (INE, 2017). Atendendo que o índice de eletrificação da Cidade de Maputo é de $100 \%$ (EDM, 2017).

\section{RESULTADOS}

\subsection{RENDA DAS FAMÍLIAS}

As diferenças de renda entre as famílias urbanas e suburbanas são bastante consideráveis. A renda média mensal de uma família urbana é aproximadamente a metade de uma família urbana. No caso do consumo mensal de eletricidade, o 
consumo médio de uma residência urbana é $280 \mathrm{KWh}$ por mês e $223.31 \mathrm{KWh}$ por mês em cada uma das residências suburbanas (inclui o consumo dos equipamentos considerados secundários). Sublinhe-se ainda que o consumo mensal de energia de cada residência de Maputo aumentou em 80KWh, comparativamente ao ano de 2016, que de acordo com a EDM (2016) o consumo mensal de cada residência era de 171 $\mathrm{KWh}$.

\subsection{OS EQUIPAMENTOS DOS CONSUMIDORES}

Nas residências urbanas são mais utilizadas as chaleiras eléctricas, os ferros de engomar, as geladeiras, as lâmpadas fluorescentes e $L E D s$, os televisores do tipo LED ou smart, enquanto as lâmpadas incandescentes, televisores de tubos de raios catódicos são mais utilizados nas famílias suburbanas. As diferenças estão relacionadas às rendas das famílias, sendo que as lâmpadas e os televisores são os equipamentos eléctricos mais utilizados tanto em residências urbanas assim como em suburbanas.

As lâmpadas incandescentes são as mais utilizadas, sem, no entanto, deixar de destacar o nível encorajador de utilização de lâmpadas fluorescentes, que a proporção da sua utilização nos bairros urbanos é de aproximadamente 4 lâmpadas por residência, e duas lâmpadas por residência nos bairros suburbanos (Quadro 1). O índice de utilização de lâmpadas $L E D$ em residências suburbanas é extremamente baixo, e nos bairros urbanos está na proporção de uma lâmpada por residência.

As lâmpadas incandescentes de $60 \mathrm{~W}$ são as mais utilizadas, principalmente em bairros suburbanos, com a proporção de duas lâmpadas por residência (Quadro 1). índice de utilização de lâmpadas incandescente acaba sendo muito preocupante, pois são utilizadas em todos os bairros de Maputo (Quadro 1). Em zonas urbanas, por exemplo, onde residem os consumidores com um poderio financeiro, as lâmpadas incandescentes de $60 \mathrm{~W}$ existem na proporção de uma unidade por residência. É também preocupante a utilização de muitas lâmpadas incandescentes de 100W nas residências suburbanas, com a proporção de uma lâmpada por residência (Quadro 1). 
E o maior índice de utilização de lâmpadas incandescentes está relacionado ao hábito de utilizar este tipo de lâmpadas, falta de informação dos tipos das lâmpadas eficientes, pouca acessibilidade das lâmpadas incandescentes nos mercados locais e o custo elevado das lâmpadas eficientes.

Em relação aos televisores, maior número de moradores, suburbanos em particular, utilizam os de tubos de raios catódicos, na proporção de uma TV por residência (Quadro 1), e maioritariamente os de 21 polegadas. Enquanto os moradores dos bairros urbanos utilizam mais os televisores construídos na base das tecnologias $L C D$, SMART, LED ou plasma.

Quanto a refrigeração, as geleiras são mais utilizadas em residências dos bairros urbanos, e congeladores em bairros suburbanos, ambos com o coeficiente de penetração abaixo de uma unidade por residência (Quadro 1).

\subsection{ELASTICIDADE DOS EQUIPAMENTOS}

Os resultados do Quadro 1 confirmam que as residências de Maputo ainda não estão saturadas, pelo que podem receber novos equipamentos. Como exemplos, temos as geladeiras, que apenas $46 \%$ das famílias é que possuem este tipo de equipamento, e as chaleiras eléctricas, com a proporção de $41 \%$ (Quadro 1). No caso das chaleiras, se o índice de penetração atingir 50\% (mais 9\%), ou seja, entrarem mais 22842 (253799 x 0.09) novas chaleiras nas famílias, com o consumo anual de $352 \mathrm{KWh}$ por família, o consumo anual pode aumentar em aproximadamente 8GWh (352 x 22842).

Por outro, o Quadro 1 apresenta as elasticidades ( $\eta$ ) que permitiram ainda estimar os efeitos de aumento na penetração de aparelhos na futura procura de electricidade em residências da Cidade de Maputo. Diga-se que um aumento de $1 \%$ de equipamentos, tais como as lâmpadas incandescentes, os televisores de raios catódicos e os refrigeradores, o consumo das famílias de Maputo pode aumentar significativamente. 
Quadro 1: Elasticidade e coeficiente de penetração dos equipamentos

\begin{tabular}{|c|c|c|c|c|c|c|c|}
\hline \multicolumn{2}{|c|}{ Equipamentos } & \multicolumn{2}{|c|}{$\begin{array}{l}\text { Consumo médio } \\
\text { mensal (KWh) }\end{array}$} & \multicolumn{2}{|l|}{$£$} & \multicolumn{2}{|c|}{$\eta(\%)$} \\
\hline & & $\mathrm{RU}$ & RS & $\mathrm{RU}$ & RS & $\mathrm{RU}$ & $\mathrm{RS}$ \\
\hline \multirow{3}{*}{ Lamp. Inc } & 40 & - & 0.1 & $\begin{array}{lll}- & -\end{array}$ & 0.008 & - & - \\
\hline & 60 & 15.2 & 24.2 & 1.0 & 2.2 & 0.06 & 0.26 \\
\hline & 100 & 11.1 & 14.9 & 0.8 & 0.8 & 0.04 & 0.06 \\
\hline \multicolumn{2}{|c|}{$\begin{array}{l}\text { Lâmpadas } \\
\text { fluorescentes }\end{array}$} & 11.3 & 7.2 & 3.7 & 2.1 & 0.16 & 0.07 \\
\hline \multicolumn{2}{|c|}{ Lâmpadas LEDs } & 2.0 & 0.7 & 1.3 & 0.4 & 0.01 & - \\
\hline \multirow[t]{2}{*}{ Televisores } & T. R.C & 8.3 & 22.4 & 0.5 & 1.3 & 0.02 & 0.14 \\
\hline & Outros & 36.9 & 12.0 & 0.9 & 0.4 & 0.12 & 0.02 \\
\hline \multicolumn{2}{|l|}{ Geladeiras } & 33.6 & 16.6 & 0.9 & 0.5 & 0.11 & 0.04 \\
\hline \multicolumn{2}{|c|}{ Congeladores } & 132 & 104.7 & 0.6 & 0.6 & 0.30 & 0.30 \\
\hline \multicolumn{2}{|c|}{ Chaleiras eléctricas } & 15.5 & 7.9 & 0.5 & 0.3 & 0.03 & 0.01 \\
\hline $\begin{array}{l}\text { Consumo } \\
\text { total } \\
\text { Residência/f }\end{array}$ & $\begin{array}{l}\text { médio } \\
\text { por } \\
\text { amília }\end{array}$ & 265.9 & 210.7 & & & & \\
\hline
\end{tabular}

Legenda: RU - Residência/família urbana; RS - Residência/família suburbanos; £ Coeficiente de penetração/média de equipamentos por residência; $\eta$ - Elasticidade; Lamp. Inc - Lâmpadas incandescentes; T.R.C - Tubos de raios catódicos.

Por exemplo, um aumento de $1 \%$ na penetração de geladeiras nas residências urbanas, de 0.9 para 0.91 , o consumo médio mensal de electricidade por família pode aumentar em $0.11 \%$, ou seja, $0.0011 \times 265.9=0.3 \mathrm{KWh}$ por mês e $3.5 \mathrm{KWh} /$ ano. Nas 108 residências urbanas (analisadas), o consumo anual pode aumentar em $379 \mathrm{KWh}$ e em toda zona urbana de Maputo (20352 residências) o consumo anual pode aumentar em 71.4MWh. 
Nesta base estima-se que um aumento de $1 \%$ do número ou penetração de televisores de tubos de raios catódicos nas famílias suburbanas, o consumo anual por família pode aumentar em 3.6KWh, 1.7MWh/ano nas 469 famílias estudadas, 0.8GWh em toda zona suburbana de Maputo. Como foi referenciado, as lâmpadas incandescentes são os equipamentos mais elásticos nas residências (Quadro 1). Em 2016, na EDM estavam registados aproximadamente 253799 clientes domésticos (EDM, 2017). Considerando que cada cliente (residência) possui pelo menos uma lâmpada incandescente de $60 \mathrm{~W}$, tal como demostram os coeficientes de penetração do Quadro 1, a entrada de 1\% deste equipamento, o consumo anual da Cidade de Maputo pode aumentar em $0.33 \mathrm{GWh} / \mathrm{ano}$.

Na perspectiva nacional, com 1346919 clientes residenciais registados na EDM em 2016, um aumento de 1\% de lâmpadas incandescentes de $60 \mathrm{~W}$ pode fazer com que o consumo anual aumente em aproximadamente 1.7GWh/ano.

Um aumento de $12 \%$ dos congeladores pode resultar em um aumento do consumo anual em 20GWh nas zonas suburbanas e 2GWh/ano em todas residências urbanas. Note-se que um aumento do número de congeladores, o consumo de electricidade aumentará significativamente apenas nas famílias suburbanas. E esta situação é possível, pois do Quadro 1 é possível observar que a penetração destes equipamentos nas zonas suburbanas é de 0.6 , e nos próximos 10 anos é possível que entrem mais congeladores.

Os resultados revelaram ainda que um aumento de 12\% de lâmpadas incandescentes de 60W nas residências suburbana, o consumo anual pode aumentar em 18GWh, na razão de $80 \mathrm{KWh} /$ ano por família, e no mínimo $0.48 \mathrm{GWh}$ /ano em todas as residências urbanas de Maputo.

Por fim, os resultados demostram que um aumento na ordem de $20 \%$ por cada tipo de televisores, o consumo mensal de Maputo pode aumentar em pelo menos 17.8GWh (Figura 1). Um aumento de 4\% de cada tipo de refrigeradores o consumo mensal da Cidade pode aumentar em 8.7GWh. O consumo mensal de Maputo pode 
ainda aumentar em 2.4GWh se o número de cada tipo de lâmpada aumentar em 1\% (Figura 1).

Figura 1: A evolução do consumo da cidade de Maputo em função da evolução dos equipamentos

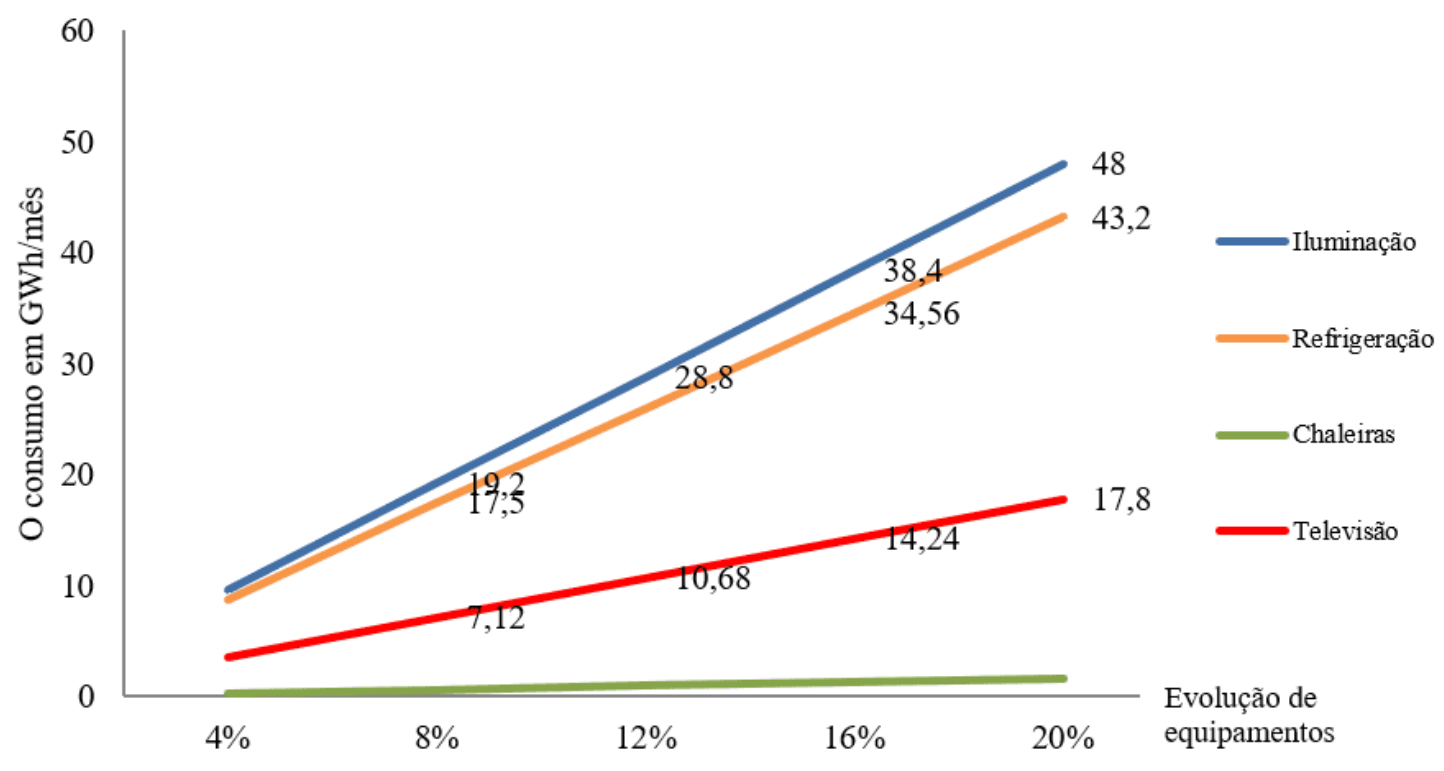

Fonte: autor.

\section{DISCUSSÃO DOS RESULTADOS}

\subsection{POLÍTICA SOBRE O PREÇO DE ELECTRICIDADE}

Embora o preço de eletricidade seja um fator importante para afetar a magnitude do consumo, esse não parece ser o caso em Maputo. Pois o aumento das tarifas preocupa mais os consumidores dos bairros suburbanos. No entanto, podem existir as razões para que isto aconteça. Por um lado porque trata-se de famílias de baixa renda e maior parte deles vivem de custas próprias, e por outro, os consumidores tendem a usar eletricidade sem racionalizar. Com isto, não deve haver apenas incentivos à poupança de eletricidade, mas também deve desincentivar o consumo de maiores quantidades deste recurso. Para desencorajar o consumo excessivo de Eletricidade, a EDM (Eletricidade de Moçambique) deve continuar a cobrar tarifas 
diferenciadas. $O$ aumento da tarifa pode variar de acordo com a quantidade de eletricidade usada pela família ou de acordo com as rendas das famílias. Esse tipo de estrutura tarifária recompensaria os consumidores com baixo consumo e desencorajaria aqueles com alto consumo. Essa medida pode forçar as categorias de alto consumo a economizarem a eletricidade.

\subsection{ADOPÇÃO DE PRÁtICAS EFICIENTES PARA A RACIONALIZAÇÃO DO USO DE ELECTRICIDADE}

Os resultados descritos nesta secção devem ser analisados, pois para o estado atender estes aumentos do consumo de eletricidade necessitará de gastar mais dinheiro, além de mais dívidas que deve contrair no exterior.

A EDM (2016), tem relatado alguns casos de défices energéticos e, maioritariamente, registados no período de $18 \mathrm{~h}$ à $22 \mathrm{~h}$, eventualmente resultantes do uso das lâmpadas e das TVs. Mesmo assim, não se pode deixar de lado que os défices podem ainda ser mais preocupantes se os outros equipamentos, tais como as geladeiras, congeladores e as chaleiras, atingirem uma penetração de pelo menos uma unidade por residência. E, para a redução do consumo, nada resta, se não utilizar racionalmente a energia, onde as ações de educação e sensibilização devem ser valorizadas.

O que encoraja é entender que o serviço de iluminação, a curto prazo, pode facilmente fornecer ganhos consideráveis ou aliviar a carga eléctrica no período de pico, bastando, para isso, trocar ou substituir as lâmpadas menos eficientes por outras mais eficientes.

A iluminação é um componente importante na procura de eletricidade em Maputo, e é responsável por 3GWh por mês (aproximadamente). A maior parte da iluminação é através de lâmpadas incandescentes (60, 100 watts). Se essas lâmpadas forem substituídas por lâmpadas fluorescentes compactas (15 watts), um aumento de 12\% destes equipamentos pode não fazer sentir muito na curva da carga da rede. 
Entretanto, o alto custo inicial das lâmpadas fluorescentes compactas (50MT por lâmpada) atua como uma grande barreira para os consumidores. E assim, a maioria dos consumidores pode não optar por essas lâmpadas. Portanto, o governo deve facilitar a produção desses aparelhos e ajudar os consumidores a substituírem as lâmpadas incandescentes por outros eficientes. E com isso o estado pode reduzir uma carga eléctrica considerável no período do pico e poupar o dinheiro que seria utilizado para novas centrais.

Note-se que distribuir 3 lâmpadas a cada cliente do país, o Estado gastaria 800 milhões de MT, mas aliviaria uma carga de 156MW no período de pico. E aliviar uma carga de 156MW significa poupar aproximadamente 19.2 bilhões de MT. Na Cidade de Maputo, o Estado gastaria 152 milhões de MT, mas aliviaria uma carga de 29MW no período de pico, e poupar aproximadamente 2 bilhões de MT, que serviria para construir uma central com esta capacidade.

\subsection{DIFUSÃO DE INFORMAÇÕES}

A educação e sensibilização sobre a eficiência dos aparelhos induziriam a poupança de energia (DIAS et al. 2004). Portanto, para que as informações cheguem à todos consumidores, as campanhas devem ser realizadas em todos os principais meios de comunicação. Trata-se de atividades que não devem ser ignoradas, pois programas potencialmente úteis e eficazes de incentivos para a racionalização do uso de eletricidade podem se tornar ineficazes devido à falta de consciencialização do consumidor (BEDIR et al. 2013). E são atividades que devem ser enquadradas nas política energéticas dos países que pretendem desenvolver de forma sustentável (BERTOLDI e REZESSY, 2006).

No mais, esses programas devem ser integrados a ferramentas de políticas que incluem programas de informação e educação e possuir regulamentos específicos de equipamentos, para que os fabricantes de aparelhos eléctricos coloquem etiquetas. Etiquetas que devem explicar a eficiência dos dispositivos e os custos operacionais estimados (REDDY, 1994). Isso permitiria que os consumidores tomassem decisões 
conscientes. E, como foi referenciado anteriormente, uma troca dos atuais equipamentos em uso por outros mais eficientes pode resultar na redução do consumo atual, e um aumento do número dos equipamentos, o aumento do consumo das famílias pode não se fazer sentir.

Para Reddy (1994), devem ser criadas páginas (de youtube por exemplo) ou uma entidade de serviço de informações sobre conservação de energia. Os serviços devem responder as perguntas do público sobre a poupança de energia e fazer sugestões, oferecendo assistência técnica a cientistas ou engenheiros para desenvolverem dispositivos eficientes. Deve também testar o desempenho de vários materiais e equipamentos de racionalização de energia.

\section{CONCLUSÕES}

No presente artigo é analisada a procura de eletricidade no sector residencial, sendo que antes foi determinado o consumo de cada aparelho residencial. Relativamente a isto, observou-se que existe uma variação considerável na quantidade de eletricidade consumida por diferentes serviços de equipamentos. Além disso, a variação das elasticidades do aparelho sugere que as respostas ou as ações de poupança de eletricidade devem, igualmente, variarem de acordos com os aparelhos. Em outras palavras, as políticas de conservação devem ser mais adaptadas ao estoque de eletrodomésticos.

No geral, as lâmpadas incandescentes desempenham um papel importante no consumo das famílias, e é importante dar a importância à redução do consumo de energia nesse serviço, através da sua substituição por outras fluorescentes ou LEDs. E uma substituição de aparelho ineficiente por eficiente a elasticidade do aparelho diminui automaticamente. Assim, os aumentos no consumo de eletricidade nas famílias de Maputo, provocados por aumento do número dos eletrodomésticos, podem ser reduzidos ou mesmo evitados. 


\section{REFERÊNCIAS}

AIGNER, D. J., SOROOSHIAN, C. and KERWIN, P. (1984). Conditional Demand Analysis for Estimating End-Use Load Profiles. Energy Journal, 81-97. Acedido no dia 3 de Novembro de 2019 em: https://www.jstor.org/stable/41321696?seq=1.

ANDERSON, K. P. (1973). Residential energy use: an econometric analysis. The Rand Corporation. Energy Conservation. Acedido no dia 22 de Julho de 2016 em: https://www.rand.org/pubs/reports/R1297.html.

ARCHIBALD, R. B., FINIFTER, D.H. and MOODY, C.E.Jr. (1982). Seasonal Variation in Residential Eletricity Demand: Evidence from Survey Data. Applied Economics,167181. Acedido no dia 3 de Novembro de 2019 em https://www.tandfonline.com/doi/abs/10.1080/00036848200000013.

BEDIR, M., HASSELAAR, E. e ITARD, L. (2013). Determinants of electricity consumption in Dutch dwellings. Energy and buildings 58, 194-207. Acedido em Maio de

em:

https://www.sciencedirect.com/science/article/abs/pii/S0378778812005257.

BERTOLDI, P. E REZESSY, S. (2006): Tradable Certificates for Energy Savings (White Certificates) - Theory and Practice. Luxembourg: Office for Official Publications of the European Communities. Acedido no dia 14 de Maio de 2019 em: https://publications.jrc.ec.europa.eu/repository/bitstream/JRC32865/2865white_cert_report_final.pdf.

BEZERRA, P. B. (2017). Proposta metodológica para avaliação da procura residencial de energia eléctrica através do desenvolvimento de uma curva de carga horária. Dissertação de Mestrado. Rio de Janeiro. Acedido no dia 28 de Junho de 2017 em: http://www.ppe.ufrj.br/images/publica\%C3\%A7\%C3\%B5es/mestrado/Paula_Borges_ da_Silvei ra_Bezerra.pdf. 
DIAS, R. A., MATTOS, C. R., E BALESTIERI, J.A.P. (2004). Energy education: breaking up the rational energy use barriers. Energy Policy volume 32. Acedido no dia 22 de Fevereiro de 2019 em: https://www.sciencedirect.com/science/article/abs/pii/S0301421503001009

DONNELY, W. A. (1984). Residential electricity demand modeling in the Australian Capital Territory: preliminary results. The Energy Journal, volume 5, 119-31. Acedido no dia 22 de Março de 2019 em https://www.iaee.org/en/publications/ejarticle.aspx?id=1642\&id=1642.

DUBIN, J.A e MCFADDEN, D.L. (1984). An econometric analysis of residential electric appliance holdings and consumption. Econométrica, volume 52: 345-362. Acedido no dia 8 de Janeiro de 2020 em: https://www.jstor.org/stable/1911493?seq=1.

EDM. (2016a). Análise do perfil de carga DTNO-B08. Maputo.

GARBACZ, C. (1983). A Model of Residential for Electricity Using a National Househoud Sample. Energy Economics, volume 5, 124 - 128. Acedido no dia 8 de Janeiro de 2020 em: https://www.sciencedirect.com/science/article/pii/0140988383900191\#!.

GARCEZ, E. W. e GHIRARDI, A. G. (2005). Elasticidades da procura residencial de energia eléctrica. Brasil. Acedido no dia 12 de Fevereiro de 2019 em: https://www.researchgate.net/publication/4731437_Elasticidades_da_Procura_Resid encial_de_Energia_Eletrica.

LAICANE, I.; BLUMBERGA, D.; BLUMBERGA; A. e ROSA, M. (2015). Evaluation of household electricity savings. Analysis of household electricity demand profile and user activities. Energy Procedia, Volume 72, 285-292. Acedido no dia 22 de Março de 2019 em: https://www.sciencedirect.com/science/article/pii/S1876610215007316.

MATTOS, L. B. e LIMA, J. E. (2005). Procura residencial de energia eléctrica em minas gerais:1970-2002. Belo Horizente. 
MOUNT, T. D., CHAPMAN, L.D., and TYRELL, T.J. (1973). Electricity Demand in the United States: An econometric analysis, Oak Ridge National Laboratory, Oak Ridge, Tennessee. Acedido no dia 22 de Julho de 2016 em:https://www.arlis.org/docs/vol2/hydropower/APA_DOC_no._1662.pdf.

PARTI, M. e PARTI, C. (1980). The total and appliance specific conditional demand for electricity in the household sector. Bell Journal of Economics. Acedido no dia 24 de Março de 2018 em: https://www.jstor.org/stable/3003415?seq=1\#page_scan_tab_contents.

REDDY, B. S. (1994). Appliance electricity consumption in the residential sector: an economic approach. Energy Sources. Acedido no dia 28 de Setembro de 2018 em https://www.tandfonline.com/doi/abs/10.1080/00908319508946077.

SILVA, A.C.M. (2017). Análise da procura condicional de energia: um instrumento para a gestão residencial. Tese de doutoramento, Brasil. Acedido no dia 16 de Marco de 2018 em http://www.din.uem.br/sbpo/sbpo2004/pdf/arq0163.pdf.

STANOVNIK, T. (1987). Appliance-specific Electricity Consumptiuon in Slovene Households. Energy Economics, volume 9, 31-36. Acedido no dia 2 de Abril de 2019 em: https://www.sciencedirect.com/science/article/pii/0140988387900041.

TAYLOR, L.D. (1975). The demand for electricity: a survey. The Bell Journal of Economics, volume 6, 74-110. Acedido no dia 16 de Marco de 2018 em: https://www.researchgate.net/publication/24048293_The_Demand_for_Electricity_A_ Survey.

WILSON, J. W. (1971). Residential demand for electricity. quarterly review of economic and business. USA. Acedido no dia 2 de Abril de 2019 em: https://books.google.co.mz/books?id=-.

ZARNIKAU, J. (2003). Functional forms in energy demand modeling. Science Direct Energy. Economics.USA. Acedido no dia 12 de Maio de 2018 em: 
https://www.frontierassoc.com/wp- content/uploads/2015/01/Final-Article.pdf ou https://www.sciencedirect.com/science/article/pii/S0140988303000434.

Enviado: Outubro, 2020.

Aprovado: Outubro, 2020. 